\title{
Fuzzy-ARTMAP based Multi-User Detection
}

\author{
Jung-Sik Lee* Regular Member
}

\begin{abstract}
This paper studies the application of a fuzzy-ARTMAP (FAM) neural network to multi-user detector (MUD) for direct sequence (DS)-code division multiple access (CDMA) system. This method shows new solution for solving the problems, such as complexity and long training, which is found when implementing the previously developed neural-basis MUDs. The proposed FAM based MUD is fast and easy to train and includes capabilities not found in other neural network approaches; a small number of parameters, no requirements for the choice of initial weights, automatic increase of hidden units, no risk of getting trapped in local minima, and the capabilities of adding new data without retraining previously trained data. In simulation studies, binary signals were generated at random in a linear channel with Gaussian noise. The performance of FAM based MUD is compared with other neural net based MUDs in terms of the bit error rate.
\end{abstract}

Key Words : Multiuser, Detector, Fuzzy-ARTMAP, Neural Network, DS-CDMA

\section{I . Introduction}

Direct-sequence CDMA is the most popular techniques. The detector receives a signal composed of the sum of all user's signals, which overlap in time and frequency. In a DS-CDMA system, the objective of the receiver is to detect the transmitted information bits of one (at mobile-end) or many (at base station) users by correlating the entire received signal with that user's code waveform.

A variety of MUD has been proposed for DS-CDMA systems. Generally, the linear minimum mean square error (MMSE) MUD is widely used, as it is computationally very simple and can readily be implemented using standard adaptive filter techniques ${ }^{[1]}$. The conventional linear detectors, however, fail to achieve good performance when channel suffers from high levels of additive noise or highly nonlinear distortion, or when the signal-to-noise ratio is poor. The linear detector can only work when the underlying noise-free signal classes are linearly separable with the introduction of proper channel delays, where the channel is assumed to be stationary. In reality, the mobile channels are going to be non -stationary where it is hard to determine the proper channel delay that varies with time. If proper channel delay is not introduced in linear MUD, the signal classes from the channel output states will be non-linearly separable.

In order to get around this problem, neural network technology has been considered in implementing MUD, because it has the capability of recovering the originally transmitted signals from nonlinear decision boundary cases $^{[2-5]}$.

Aazhang et al. $^{[2]}$ first reported a study of multi-layer perceptrons (MLP) in CDMA systems, and showed that its performance is close to that of the optimum receiver in both synchronous and asynchronous Gaussian channels. Although the simulation results proved that back-propagation learning rule outperforms the conventional one, it still leaves a lot of difficulties, such as long training time, performance sensitivity over network parameters including initial weights, and finding the proper number of hidden layer and hidden nodes.

\footnotetext{
※ This paper was supported by research funds of Kunsan National University.

* Dept. of Information \& Telecommunication Eng., Kunsan National University (leejs@kunsan.ac.kr) 논문번호 : KICS2012-01-031, 접수일자 : 2012년 1월 27일, 최종논문접수일자 : 2012년 3월 20일
} 
Also, Mitra and Poor ${ }^{[3]}$ applied a RBF network to the MUD problem. The simulation results show that the RBF based MUD is its intimate link with the optimal one-shot detector, and its training times are better and more predictable than the MLP based MUD. However, the RBF based MUD obviously requires more RBF centers, when both channel order and the number of users increase. Some researchers applied support vector machines ${ }^{[6]}(\mathrm{SVM})$ to Multi-user detecton techniques ${ }^{[7]}$. Although SVM based MUD closely match the performance of the optimal Bayesian detector, requiring a relatively small training data set, it still lead to larger model size, in comparison with the number of noise free signal states.

Since the advent of the fuzzy-ARTMAP (FAM) neural networks, the application of FAM has been discussed mainly as it applies to pattern recognition fields. Lee et al. ${ }^{[8]}$ applied FAM neural networks to channel equalization problems where it shows that its performance is comparable to RBF based equalizer with high speed. In this paper, a FAM neural network is applied to multi-user detection problem in the digital communication systems. The main purpose of the proposed FAM based MUD is to overcome the obstacles in implementing the previously developed neural-basis MUDs above.

The proposed FAM based MUD provides attractive characteristics that are not found in previously developed neural-basis MUDs; a small number of parameters, fast and easy training, no requirement for the choice of initial weights, automatic increase of hidden units, and capability of adding new data without retraining previous patterns.

Section II presents a brief summary of FAM network and learning mechanism. Section III gives the structure and learning procedure for the FAM based MUD. Simulation results are provided in Section IV, and Section V gives the conclusions.

\section{Background of Fuzzy-ARTMAP Neural Network}

Since the advent of ART (adaptive resonance theory) as a cognitive and neural theory ${ }^{[9]}$, a number of ART neural network architectures have been progressively developed. Recently, a growing number of models computationally synthesize properties of neural networks, and fuzzy logic. Fuzzy-ARTMAP (FAM) is one such model, combined with ARTMAP and fuzzy logic ${ }^{[10]}$. A schematic diagram of FAM network is shown in Fig.1.

The FAM utilizes a minimax learning rule that conjointly minimizes prediction error and maximizes generalization. As learning proceeds, the input and stored prototype of a category are said to resonate when they are sufficiently similar. When an input pattern is not sufficiently similar to any existing prototype, a new node (or hidden unit) is created to represent a new category with the input patterns as the prototype. The meaning of similarity depends on

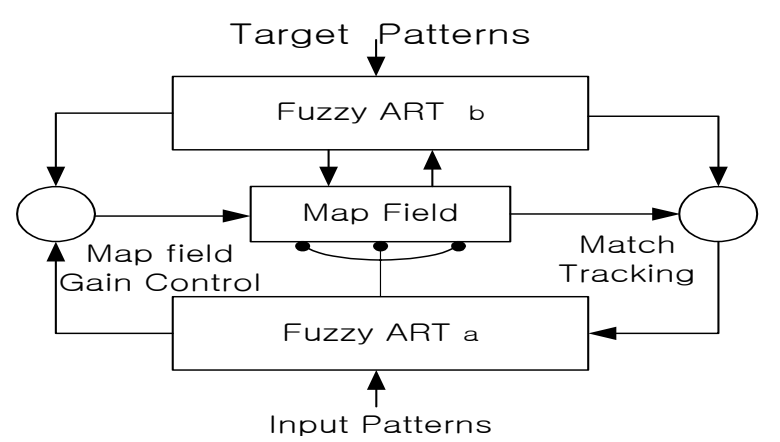

Fig. 1. Structure block diagram of FAM

a vigilance parameter $\rho$, with $0<\rho \leq 1$. If $\rho$ is small, the similarity condition is easier to meet, resulting in a coarse categorization. On the other hand, if $\rho$ is set close to 1 , many finely divided categories are formed. As a result, a FAM system increases the network architectures (number of clusters)to the minimum level necessary for perfect performance on the training data. By selecting the desired level for the vigilance parameter, the user has control over the performance of the work. For the details of the training algorithms, the readers are referred to see the paper ${ }^{[10]}$.

\section{Implementation of the FAM based MUD}


Fig. 2 shows the discrete time model of a FAM based synchronous DS-CDMA communication

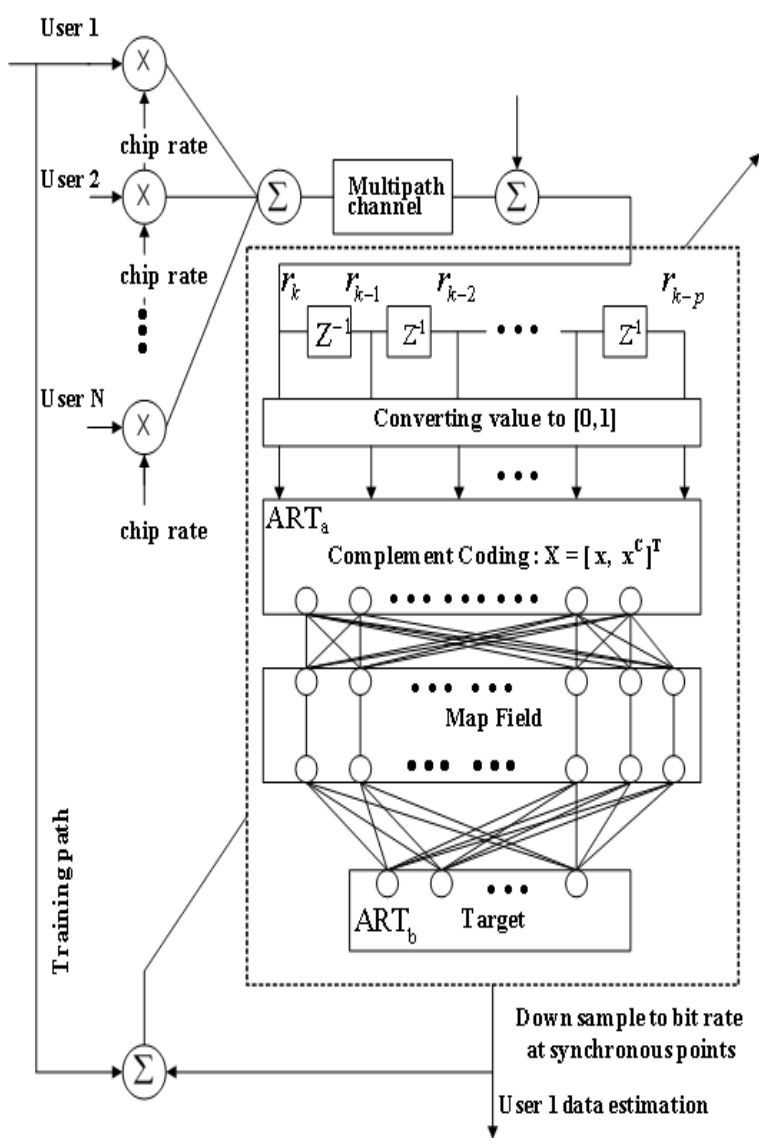

Fig. 2. Structure of FAM based MUD

supporting $N$ users with $M$ chips. The data bit $s_{i, k} \in\{ \pm 1\}$ denotes the symbol of user $i$ at time $k$, which is multiplied by the spreading, or signature waveform where $u_{i}$ is the chip wave form with unit energy.

The signature sequence for user $i$ is represented as

$$
\mathbf{u}_{i}=\left[u_{i, 1}, \ldots, u_{i, M}\right]^{T}
$$

and the channel impulse response is

$$
H(z)=h_{0}+h_{1} z^{-1}+\ldots+h_{q} z^{-q}
$$

where $q$ denotes the channel order. The baseband model for received signal sampled at chip rate is represented as [4].

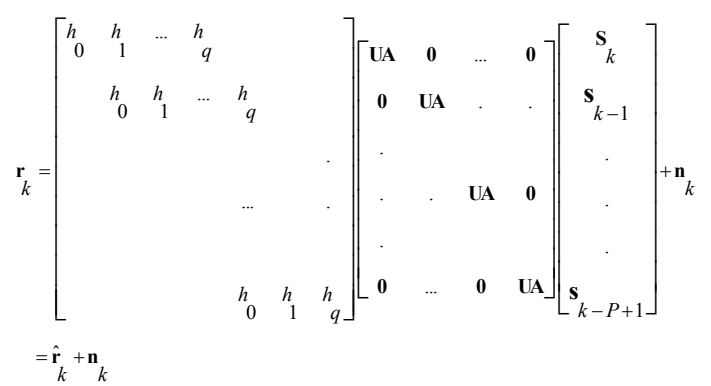

where the user symbol vector $\mathbf{S}_{k}=\left[s_{1, k}, s_{2, k}, \ldots, s_{N, k}\right]^{T}$, the white Gaussian noise vector $\mathbf{n}_{k}=\left[n_{1, k}, \ldots, n_{M, k}\right]^{T}, \quad \hat{\mathbf{r}}_{k \text { denotes the }}$ noise free received signal. The first, second, and third part of ${ }_{k}^{\mathbf{r}}$ are $M \times P M$ channel impulse response matrix, $P M \times P N$, and $P N \times 1$, respectively ${ }^{[4]}$. Thus, the ${ }_{k}{ }_{k}$ is the $M \times 1$ vector. $\mathbf{U}=\left[\mathbf{u}_{1}, \ldots, \mathbf{u}_{N}\right]$ denotes the normalized user code matrix, and the diagonal user signal amplitude matrix is given by $\mathbf{A}=\operatorname{diag}\left\{A_{1}, \ldots, A_{N}\right\}$. The channel inter symbol interference span $P$ depends on the channel order $q$ and the chip sequence length $M: \quad P=1 \quad$ for $\quad q=0, \quad P=2$ for $0<q \leq M-1, \quad P=3 \quad$ for, $M-1<q \leq 2 M-1$ and so on.

\subsection{Adaptive Training of FAM based MUD}

Considering the third part in (3), the user symbol vectors, $\mathbf{S}_{k}$, the number of user, $N$ and the number of interference span, $P$, there are $N_{s}=2^{N P}$ possible combinations of the channel input sequence. Here $\mathbf{S}_{k}^{j}$ is represented as

$$
\mathbf{S}_{k}^{j}=\left[\mathbf{s}_{k}^{j}, \mathbf{s}_{k-1}^{j}, \mathbf{s}_{k-2}^{j}, \ldots, \mathbf{s}_{k-P+1}^{j}\right]^{T}, 1 \leq j \leq N
$$

This produces $2^{N P}$ values of the noise-free channel 
output vector

$$
\mathbf{r}_{k}=\left[r_{k}, r_{k-1}, \ldots, r_{k-M+1}\right]^{T}
$$

These vectors will be referred to as the desired channel states, and they can be partitioned into two classes according to the corresponding value in

$\mathbf{S}_{k}^{j}$, depending on which user is considered in making decision (here no channel delay is assumed

$$
\left.\begin{array}{l}
R_{i}^{+}=\left\{\hat{r}_{k} \mid s_{i, k}=1\right\}, \\
R_{i}^{-}=\left\{\hat{r}_{k} \mid s_{i, k}=-1\right\},
\end{array}\right\}
$$

In order to train a neural network to serve as a multi-user detector, it is necessary to generate appropriate training data. In this study, the network is trained to reconstruct the original signal( 1 or -1$)$ based on the signal received after transmission over a dispersive channel, as shown in Fig. 2. Therefore, input patterns for the network consist of received signals and the corresponding target is the original transmitted signal. If pure training patterns were available, they could be used directly, but if FAM is to be trained with noisy signals, preprocessing is necessary to prevent the network from learning the noise. In this study, the situation of a noisy transmission path is simulated by adding Gaussian noise to the received signal after each possible transmission sequence is passed through the transfer function. The training patterns are generated by applying the supervised K-means clustering algorithm $^{[5]}$ to remove the Gaussian noise.

The training patterns which come from the transfer function (either directly or after the noise removal) have components that are not in the correct range for fuzzy-ARTMAP. The actual range depends on the transfer function; however, the binary sigmoid

$$
\frac{1}{1+e^{-\alpha x}}
$$

converts the interval $[-n, n]$ to $[0,1]$, and thus is suitable for making the required conversion for any transfer function. The final input vectors after converting and complement coding procedures are

$$
\mathbf{X}_{i}=\left[\mathbf{x}_{i}, \mathbf{x}_{i}^{c}\right]^{T}, i=1,2, \ldots, N_{s}
$$

where

$$
\begin{gathered}
\mathbf{x}_{i}=\left[x_{i 0}, x_{i 1}, \ldots, x_{i p}\right] \\
\mathbf{x}_{i}^{c}=\left[1-x_{i 0}, 1-x_{i 1}, \ldots, 1-x_{i p}\right] \\
x_{i j}=\frac{1}{1+\exp \left(-\alpha \cdot r_{i j}\right)}, j=0,1, \ldots, p
\end{gathered}
$$

where $\quad r_{i j}$ is the estimated training input component from noisy input signals by using $\mathrm{K}$-means clustering algorithm ${ }^{[5]}$. The target value for each generated training pattern is the correct value for $s_{d, k}$ for the desired delay, $d$. The appropriate value of $d$ is determined by the dominant term in the transfer function. A target value of 1 is represented by the vector $(1,0)$; the target value of 1 is given by the vector $(0,1)$. To generate training patterns for a given channel input matrix and noisy channel output vector, it is necessary to estimate the channel order. This is done using regression analysis ${ }^{[5]}$. For details of overall training algorithms, readers are referred to see the paper $^{[10]}$.

\section{Simulation Studies}

The performance of FAM neural network was evaluated in terms of pattern recognition capabilities, while comparing with other neural networks such as multilayer perceptron and radial basis functions nerual networks. Figure 3 show the performance comparison of three different neural networks. The eight data patterns in the graph were arbitrarily chosen. As shown in graph, the response regions (shaded area) of radial basis functions and 
fuzzy-ARTMAP are better than that of multilayer

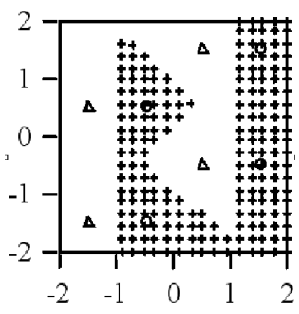

(a)

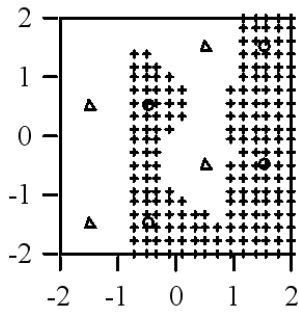

(c)

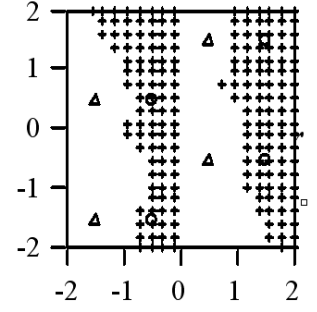

(b)

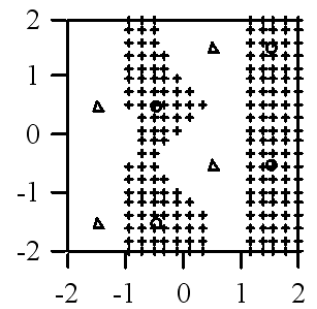

(d)

(a) Ideal (b) MLP (c) RBF (d) Fuzzy-ARTMAP

Fig. 3. Comparison of nonlinear decision boundary

perceptrons (MLP). In the training of MLP, the number of units used in input, hidden, and output layers were two, eight, and one, respectively. The RBF neural networks uses eight centers. For the training of fuzzy-ARTMAP, the vigilance, and sigmoid steepness parameters were 0.95 , and 0.85 respectively. Fig. 4 shows the effect of vigilance value in training fuzzy-ARTMAP neural networks ${ }^{[8]}$.

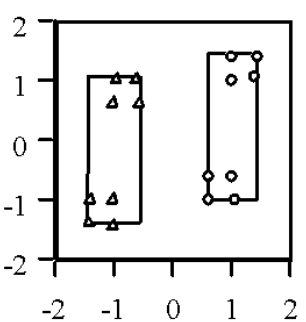

(a) vigilance parameter $=0.7,2$ clusters

(b) vigilance parameter $=0.85,4$ clusters

Fig. 4 The effect of vigilance parameter with 16 patterns (8 circles, 8 triangles)

As shown in Fig. 4, high vigilance results in more clusters, and the performance with high vigilance was better than with low vigilance. The vigilance value also affects the region which each cluster will accept when the net is applied (after training). The cluster will accept all points that fall within the boundaries shown, and also points somewhat outside the dotted rectangle region.

Simulation studies were performed to compare different neural networks based MUD. For the purpose of showing that multiuser detection can be regarded as a classification problem, a very simple two user system with 2 chips per symbol was considered. The chip sequences of the two users were set as $(-1,-1)$ and $(-1,1)$, respectively. The following are channel impulse responses used in simulation

$$
\left.\begin{array}{l}
H_{1}(z)=1+0.4 z^{-1} \\
H_{2}(z)=0.8+0.5 z^{-1}+0.3 z^{-2}
\end{array}\right\}
$$

The two users are assumed to have equal signal power. Simulation works consist of some procedures. The first is to estimate both noise free received signals and noise variances using supervised $k$-means clustering ${ }^{[5]}$. The next one is to train the estimated signal using fuzzy-ARTMAP neural networks.

Fig. 5 shows the error rate comparison of one linear and four kinds of neural network based MUD over two different channel models. The bit error rate (BER) performance was conducted with 100,000 inputs with Gaussian noise. In MLP based MUD, the number of units used in input, hidden, and output layers were two, eight, and one, respectively. The RBF MUD uses four centers for the case of $H_{1}(z)$, eight centers for $H_{2}(z)$ in (17). In the simulation studies of SVM based MUD, Gaussian functions were used as kernels, where six support vectors were selected with controlling parameter, $\mathrm{C}=5$, for $H_{1}(z)$ and four support vectors with parameter, $\mathrm{C}=10$, for $H_{2}(z)$. In the training of fuzzy-ARTMAP based MUD, the vigilance value was 0.8 , resulting in four categories for $H_{1}(z)$ while 0.9 value of vigilance parameter with eight 
categories for $H_{2}(z)$. The steepness parameter for

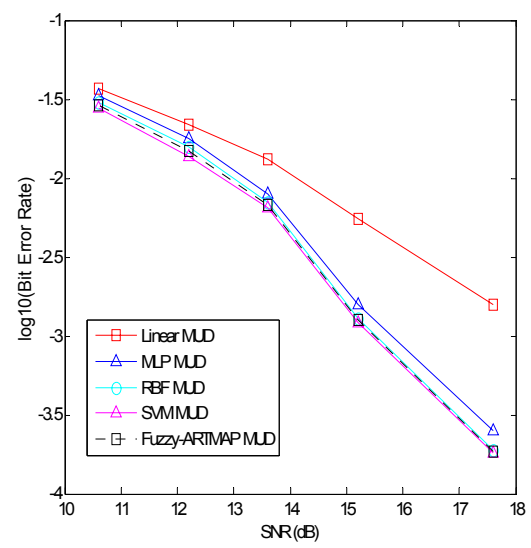

(a)

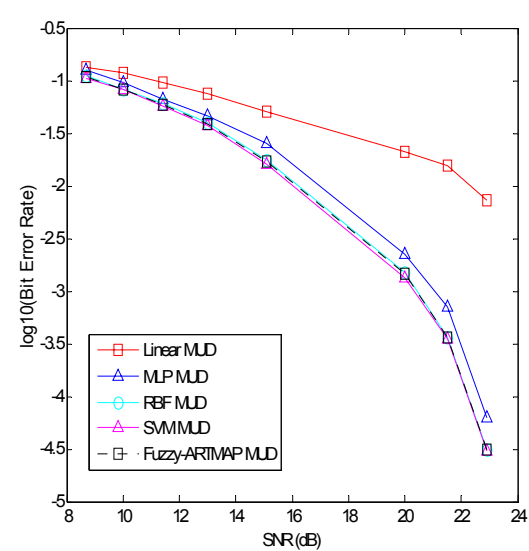

(b)

(a) $H(z)=1+0.4 z^{-1}$

(b) $H(z)=0.8+0.5 z^{-1}+0.3 z^{-2}$

Fig. 5. A comparison of error rate performance

transfer function was selected as the same value as noise variance estimated from k-means clustering techniques for both channel types. As shown in the graph, the performance of the FAM based MUD is superior to that of the linear MUD and better than MLP based MUD, while producing results as favorable as those in both RBF and SVM based MUDs.

\section{Conclusion}

In the paper, the FAM based MUD was developed and compared with the MUDs based on other types of neural networks, such as MLP, RBF and SVM. Although the performance of the both RBF and SVM based MUDs are almost the same as that of the FAM based MUD, the FAM based MUD is a more viable candidate than the RBF and SVM based MUDs, considering the following cost and efforts required in neural network implementation; a small number of parameters, no requirements for the choice of initial weights, automatic increase of hidden units, no risk of getting trapped in local minima, and the capabilities of adding new data without remaining previously trained data. In addition, the training speed of the FAM based MUD over the MLP, RBF, and SVM based MUDs can be much faster as the order of channel impulse response increases.

Research has been continuing into more complex cases with higher channel order, many users, and long chip sequences. The modified or simplified form of FAM training technique and its application to MUD will be investigated and analyzed.

\section{References}

[1] Poor, H.V.,Verdu, S., "Probability of errors in MMSE multiuser detection," IEEE Transactions on Information Theory, Vol. 43, pp. 858-871, 1997.

[2] Aazhang, B., Paris, B.P., Orsak, G.C., "Neural networks for multiuser detection in code-division multiple-access channels," IEEE Transactions on Communications, Vol. 40, pp. 1212-1222, 1992.

[3] Mitra, U., Poor, H.V., "Neural network techniques for adaptive multiuser demodulation," IEEE Journal on Selected Areas in Communications, Vol. 12, pp. 1460-1470, 1994.

[4] Chen, S., Samingan, A. K., Hanzo, L., "Adaptive near minimum error rate training for neural networks with application to multiuser detection in CDMA communication systems," Signal Processing, Vol. 85, pp. 1435-1448, 2005.

[5] Chen, S., Mulgrew, B., Grant, P.M., "A clustering technique for digital communication 
channel equalization using radial basis function networks," IEEE Transactions on Neural Networks, Vol. 4, pp. 570-579, 1993.

[6] Burges, C. C. "A tutorial on support vector machine for pattern recognition," Data Mining and Knowledge Discovery, vol. 2, pp. 121-167, 1998.

[7] Chen, S., Samingan, A. K., Hanzo, L., "Support vector machine multiuser receiver for DS-CDMA signals in multipath channels", IEEE Trans. on Neural Networks, vol. 12, pp. 604-611, 2001.

[8] Lee, J., Choi, Y., Lee, J., Han, S., "Channel equalization using fuzzy ARTMAP," IEICE Transactions on Communication, Vol. E85-B, pp. 826-830, 2002.

[9] Grossberg, S., "Adaptive pattern classification and universal recording: I. Parallel development and coding of neural feature detectors," Biological Cybernetics , pp. 121-134, 1976.

[10] Carpenter, G., Grossberg, S., Markuzon, N., Reynolds, J., Rosen, D., "Fuzzy ARTMAP: A neural network architecture for incremental supervised learning of analog multidimensional maps," IEEE Trans. Neural Networks, Vol. 3, pp. 698-713, Sep. 1992.
이 정 식 (Jung-Sik Lee) 정회원

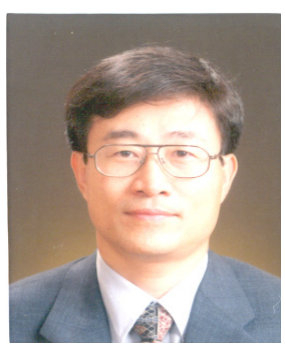

1983년 한양대학교 전자통신과 공학사

1990년 Florida Institute of Tech., Elec. Eng. M.S.

1996년 Florida Institute of Tech., Elec. Eng. Ph.D. 1997년 현재 군산대 정보통신 공학과 정교수

2004년 2006년 미국 남부 플로리다 주립대

(Univ. Of South Florida) 방문 교수 1986년 1988년 OPC 중앙연구소 연구원 <주관심분야> 적응신호처리,디지털 통신, 신경망응 용 